\title{
Spectrum of Wind Power Fluctuations
}

\author{
M. M. Bandi* \\ Collective Interactions Unit, OIST Graduate University, Okinawa 904-0495, Japan
}

(Received 15 September 2016; published 13 January 2017)

\begin{abstract}
Wind power fluctuations for an individual turbine and plant have been widely reported to follow the Kolmogorov spectrum of atmospheric turbulence; both vary with a fluctuation time scale $\tau$ as $\tau^{2 / 3}$. Yet, this scaling has not been explained through turbulence theory. Using turbines as probes of turbulence, we show the $\tau^{2 / 3}$ scaling results from a large scale influence of atmospheric turbulence. Owing to this long-range influence spanning 100s of kilometers, when power from geographically distributed wind plants is summed into aggregate power at the grid, fluctuations average (geographic smoothing) and their scaling steepens from $\tau^{2 / 3} \rightarrow \tau^{4 / 3}$, beyond which further smoothing is not possible. Our analysis demonstrates grids have already reached this $\tau^{4 / 3}$ spectral limit to geographic smoothing.
\end{abstract}

DOI: 10.1103/PhysRevLett.118.028301

All renewables fluctuate with the natural variability in their energy sources [1,2]. Wind [3] and solar photovoltaics [4], in particular, exhibit fluctuations over a range of magnitudes and time scales (from milliseconds up to a day). Such fluctuations threaten electrical grid stability $[5,6]$ when their magnitudes form a large fraction of power carried by the grid over time scales comparable to the grid response time. Consequently, grid integrity demands increased ancillary reserves, in turn adding to the cost of renewables [7]. In addition, dynamically balancing the ever present consumer load fluctuations with the variable power output of renewables becomes very challenging [8]. Naturally, understanding fluctuations in renewables is of great import in designing strategies to mitigate or manage their fluctuations. Increasing penetration of renewables within the global energy mix also renders this problem very timely and relevant.

The power generated by a wind turbine fluctuates with varying wind speed and indeed, its spectrum is widely believed to reflect the Kolmogorov spectrum [9] of atmospheric turbulence; both vary with frequency $f$ as $f^{-5 / 3}$ [10-12] or with time scale $\tau$ as $\tau^{2 / 3}$ [13]. This variability decreases when fluctuations are averaged in the aggregate power feeding the grid from geographically distributed wind plants [14], a mechanism referred to as geographic smoothing. Despite costs associated with variability [15], neither the $\tau^{2 / 3}$ fluctuation scaling nor the geographic smoothing mechanism are understood.

We provide a minimal description of the wind power fluctuation spectrum from the turbine through grid scales from a turbulence theory standpoint and experimentally

Published by the American Physical Society under the terms of the Creative Commons Attribution 3.0 License. Further distribution of this work must maintain attribution to the author(s) and the published article's title, journal citation, and DOI. verify it with wind plant data. The $\tau^{2 / 3}$ wind power fluctuation scaling results from the largest length scales of atmospheric turbulence spanning hundreds of kilometers, influencing the small scales where individual turbines operate. This longrange influence correlates power outputs from geographically distributed plants over a range of time scales that falls with interplant distance. Consequently, aggregate grid-scale power fluctuations smooth until they reach a limiting spectrum with $\tau^{4 / 3}$ scaling.

The power $P$ generated by a wind turbine relates to wind speed $u$ blowing past the turbine through the power relation $P=\left(\frac{1}{2}\right) \rho A \gamma u^{3}$. Here, $\rho$ is air density, $A$ is the turbine rotor cross sectional area, and $\gamma$ is the turbine efficiency. This dimensional argument can be expressed in time-dependent form as (Supplemental Material [16])

$$
P(t)=K\left[\bar{u}^{3}+3 \bar{u}^{2} \tilde{u}(t)+3 \bar{u} \tilde{u}(t)^{2}+\tilde{u}(t)^{3}\right]
$$

where $K=\left(\frac{1}{2}\right) \rho A \gamma, \bar{u}$ is the time-independent mean, and $\tilde{u}(t)$ is the time-varying fluctuation in speed $[\overline{\tilde{u}(t)}=0]$. We analyze wind power fluctuations from a turbulence standpoint by treating the turbine as a temporal, Eulerian probe, much like an anemometer, whose output is a function of time-varying turbulent wind speed past a fixed (Eulerian) spatial point (Supplemental Material [16]). Assuming the turbine extracts the same fraction of power at all frequencies, i.e., no dispersion and that the power conversion is adiabatic, characteristics of Eulerian, temporal fluctuations in atmospheric turbulence apply to wind power alike. This idealized assumption fails at time scales $\tau \leq \tau_{R}$ [11], where $\tau_{R} \sim$ tens of seconds is the turbine reaction time scale, but holds at long time scales $\left(\tau_{R}<\tau<\tau_{0}\right)$ until velocity fluctuations decorrelate at the large eddy turnover time $\tau_{0}$ (Table I).

We deduce the second order structure function for wind power in terms of wind speed by substituting Eq. (1) in 
TABLE I. Definitions of parameters and variables.

\begin{tabular}{lc}
\hline \hline Quantity & Description \\
\hline$l_{0}$ & Integral length scale \\
$\tau_{0}$ & Large eddy turnover time \\
$\eta$ & Kolmogorov length scale \\
$\bar{\varepsilon}$ & Mean energy flux per unit mass \\
$\tilde{u}_{\|}(\vec{r})$ & Longitudinal velocity at coordinate $\vec{r}$ \\
$\Delta u_{\|}^{m}(r)$ & {$\left[\tilde{u}_{\|}(\vec{R}+\vec{r})^{m}-\tilde{u}_{\|}(\vec{R})^{m}\right]$} \\
$\Delta u^{m}(\tau)$ & {$\left[\tilde{u}(t+\tau)^{m}-\tilde{u}(t)^{m}\right]$} \\
$S_{n}^{m}(r)$ & $\left\langle\left[\Delta u_{\|}^{m}(r)\right]^{n}\right\rangle$ \\
$S_{n}^{m}(\tau)$ & $\left\langle\left[\Delta u^{m}(\tau)\right]^{n}\right\rangle$ \\
$u_{\mathrm{rms}}$ & {$[P(t+\tau)-P(t)]$} \\
$\Delta P(\tau)$ & $\left\langle[\Delta P(\tau)]^{2}\right\rangle$ \\
$D_{2}(\tau)$ & $\mathrm{rms}$ velocity $\sqrt{\tilde{u}^{2}} \equiv \sqrt{\left\langle\left[\Delta u\left(\tau_{0}\right)\right]^{2}\right\rangle}$. \\
\hline \hline
\end{tabular}

$\Delta P(\tau)$ (Table I) and computing $D_{2}(\tau)=\left\langle\left\{K\left[3 \bar{u}^{2} \Delta u^{1}(\tau)+\right.\right.\right.$ $\left.\left.\left.3 \bar{u} \Delta u^{2}(\tau)+\Delta u^{3}(\tau)\right]\right\}^{2}\right\rangle$, obtaining to leading order (ignoring higher order cross terms)

$$
D_{2}(\tau) \sim 9 K^{2} \bar{u}^{4} S_{2}^{1}(\tau)+9 K^{2} \bar{u}^{2} S_{2}^{2}(\tau)+K^{2} S_{2}^{3}(\tau) .
$$

Equation (2) now affords us an interpretation of the wind power fluctuation spectrum through the framework of turbulence theory. We analyzed scalings for all terms in Eq. 2 with data from a 2.05 MW MM92 REPower turbine in Howard, NY. The simultaneous wind speed and power time series sampled at $0.2 \mathrm{~Hz}$ for 20 day duration had mean speed $\bar{u}=8.04 \mathrm{~m} \mathrm{~s}^{-1}$ and rms fluctuation $u_{r m s}=4.58 \mathrm{~m} \mathrm{~s}^{-1}$. Autocorrelation functions [Fig. 1(a)] yielded a large eddy turnover time $\tau_{0}=13 \mathrm{~h}$ for wind speed and decorrelation time of $14 \mathrm{~h}$ for power, clearly pointing to the strong influence of diurnal oscillations on signal correlation.

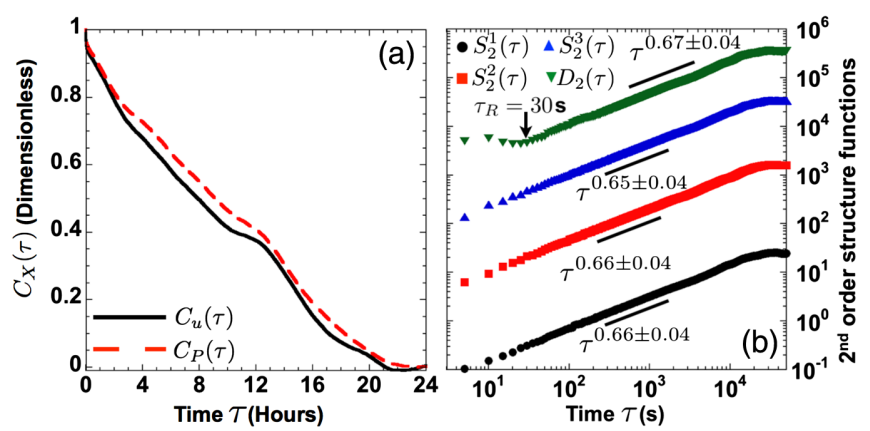

FIG. 1. (a) Autocorrelation functions $\left[C_{X}(\tau)=[\overline{X(t) X(t+\tau)} /\right.$ $\overline{X(t)^{2}}$, where $X(t)=\tilde{u}(t)$ for wind speed and $P(t)-\bar{P}$ for wind power] exhibit correlation time of $\tau_{0}=13 \mathrm{~h}$ (large eddy turnover time) for wind speed (solid black line) and $14 \mathrm{~h}$ for wind power (dashed red line). (b) $S_{2}^{1}(\tau)$ (solid black circles), $S_{2}^{2}(\tau)$ (solid red squares), $S_{2}^{3}(\tau)$ (solid blue triangles) representing rhs and $D_{2}(\tau)$ (solid green inverted triangles), the lhs of Eq. (2), all scale as $\tau^{2 / 3}$ within measurement error $( \pm 0.04) . D_{2}(\tau) \sim \tau^{2 / 3}$ scaling deviates for $\tau \leq \tau_{R}=30 \mathrm{~s}$.
Figure 1(b) plots all terms in Eq. (2) for Howard data. Barring deviations at short time scales $\left(\tau \leq \tau_{R} \simeq 30 \mathrm{~s}\right)$ expected from rotor inertia, $D_{2}(\tau) \sim \tau^{0.67 \pm 0.04}$ agrees with prior empirical results [10-12]. All $S_{2}^{m}(\tau) \sim \tau^{2 / 3}$ in Eq. (2) to within measurement error, in accord with atmospheric turbulence measurements [35] in the absence of turbines. We now explain this $\tau^{2 / 3}$ scaling through turbulence theory.

Turbulent kinetic energy is transported in fluid parcels, loosely termed "eddies", of finite spatial extent over which velocity fluctuations are correlated. When transported past a stationary, Eulerian probe, an eddy registers a fluctuation of finite duration. Fluctuations therefore represent the length $r$ and time $\tau$ scales within the inertial range of turbulence $\eta<r<l_{0}$ (Table I). Kolmogorov's 1941 theory (K41) [9] elucidates the velocity $\left(\tilde{u}^{1}\right)$ fluctuation spectrum within the inertial range of fully developed (high Reynolds $R e$ ) three dimensional turbulence through the second order structure function $S_{2}^{1}(r)=C_{1}(\bar{\varepsilon} r)^{2 / 3}\left[C_{1} \sim \mathcal{O}(1)\right.$ constant]. Since we are concerned with temporal wind power fluctuations, Taylor's hypothesis (TH) [36] permits a convenient [37] switch from length to time scales $r \equiv \bar{u} \tau$ to retrieve the Eulerian, temporal second order structure function $S_{2}^{1}(\tau)=C_{1}(\bar{u} \bar{\varepsilon} \tau)^{2 / 3}$. Whereas K41 concerns only velocity fluctuations $\left[S_{2}^{1}(\tau)\right]$, Eq. (2) contains additional terms $S_{2}^{2}(\tau)$ and $S_{2}^{3}(\tau)$.

Dimensional analysis similar to K41 extended to arbitrary powers of velocity yields $S_{n}^{m}(r) \sim(\bar{\varepsilon} r)^{2 m / 3}$ (Supplemental Material [16]), but substituting $r \equiv \bar{u} \tau$ does not convert these expressions from space to time. This conversion depends on the relative magnitude of mean speed to rms fluctuations $\left(u_{\mathrm{rms}} / \bar{u}\right)$. When $u_{\mathrm{rms}} / \bar{u} \ll 1$, (operationally $u_{\mathrm{rms}} / \bar{u}<0.1$ ), the uniform sweeping regime arises where TH applies. Here, eddies do not measurably evolve over measurement duration as they are swept past the Eulerian probe by $\bar{u}$, thus yielding

$$
\begin{array}{rl}
D_{2}(\tau) \sim 9 & 9 K^{2} \bar{u}^{14 / 3}(\bar{\varepsilon} \tau)^{2 / 3}+9 K^{2} \bar{u}^{8 / 3} u_{\mathrm{rms}}^{2}(\bar{\varepsilon} \tau)^{2 / 3} \\
& +K^{2} u_{\mathrm{rms}}^{4}(\bar{u} \bar{\varepsilon} \tau)^{2 / 3} .
\end{array}
$$

$S_{2}^{2}(\tau) \& S_{2}^{3}(\tau)$ scalings differ from K41 dimensional expectations due to breakdown of Galilean invariance (Supplemental Material [16]). When $u_{\mathrm{rms}} / \bar{u}>0.1$, the random sweeping hypothesis (RSH) applies and accounts for eddy evolution over measurement duration, yielding

$$
\begin{aligned}
D_{2}(\tau) \sim & 9 K^{2} \bar{u}^{4}\left(u_{\mathrm{rms}} \bar{\varepsilon} \tau\right)^{2 / 3}+9 K^{2} \bar{u}^{2} u_{\mathrm{rms}}^{8 / 3}(\bar{\varepsilon} \tau)^{2 / 3} \\
& +K^{2} u_{\mathrm{rms}}^{14 / 3}(\bar{\varepsilon} \tau)^{2 / 3}
\end{aligned}
$$

The left-hand side (lhs) of Eqs. (3) and (4) will exhibit $D_{2}(\tau) \sim \tau^{2 / 3}$ scaling over the range of time scales $\tau_{R}<\tau<\tau_{0}$. For $\tau \leq \tau_{R}, D_{2}(\tau)$, scaling will deviate due to turbine inertia and control system dynamics, but $S_{2}^{1}(\tau)$, $S_{2}^{2}(\tau)$, and $S_{2}^{3}(\tau)$ scalings on right-hand side (rhs) of 
Eqs. (3) and (4) will persist. As $\tau \rightarrow \tau_{0}$, all scalings will deviate from $\tau^{2 / 3}$ scalings due to loss of correlation. All these features are observed in Fig. 1(b). Whereas $u_{\mathrm{rms}} / \bar{u} \simeq$ 0.57 places Howard data [Fig. 1(b)] within the RSH regime (Eq. (4)), TH and RSH in fact represent two limits. In reality, both $\bar{u}$ and $u_{\text {rms }}$ participate in eddy transport; hence, it requires the application of a combination of $\mathrm{TH}$ and $\mathrm{RSH}$ $[38,39]$. Nonetheless, this has no impact on the $\tau^{2 / 3}$ scaling since both Eqs. (3) and (4) yield the same exponent.

To our knowledge, this is the first physical explanation of the wind turbine power fluctuation spectrum. The presence of $u_{\text {rms }}$ in $D_{2}(\tau)$ implies power fluctuations in an individual turbine contain signatures of the largest scales of atmospheric turbulence. Furthermore, two turbines distance $d \leq l_{0}$ apart exhibit correlated power fluctuations over a range of time scales that varies with $d$. This is owing to $u_{\mathrm{rms}}$ being the integral scale $\left(l_{0}\right)$ velocity fluctuation, through which the largest scales of atmospheric turbulence exert influence upon individual turbines. An estimate of the integral scale from Howard data yields $l_{0}=u_{\mathrm{rms}} \tau_{0}=4.58 \mathrm{~ms}^{-1} \times 13 \mathrm{~h}=214 \mathrm{~km}$, clearly, a consequence of diurnal oscillation time scale dominating over the long signal correlation time (Supplemental Material [16]). This estimate is in good agreement with independent studies that directly measured interplant, instantaneous (zero time lag, $\tau=0$ ) power fluctuation correlations which fell with distance. This decay in correlation was fit with an exponential $\left[e^{(-d / D)}\right]$ providing a correlation decay length $D \sim$ hundreds of km; $D=641 \mathrm{~km}$ (Europe) [40], $D=305 \mathrm{~km}$ (Electric Reliability Council Of Texas ERCOT system, Texas) [14], $D=375 \mathrm{~km}$ (Germany) [41], and $D=500 \mathrm{~km}$ (Denmark) [42]. Henceforth, we assume $l_{0} \simeq D$ in all subsequent analysis.

We now consider aggregate power fluctuations in a wind plant comprising, say $M$ turbines. If individual turbines were uncorrelated, aggregate plant fluctuations would smooth as $1 / \sqrt{M}$, and the spectrum would continually steepen $\tau^{\zeta}$ (with $\zeta>2 / 3$ ) with increasing $M$. Such is not the case; wind plant power fluctuations also exhibit $\tau^{2 / 3}$ scaling $[10,12,14]$. At least three sources of turbulence must be considered at the plant level - turbulence generated by the atmospheric boundary layer (boundary layer dynamics), collective distortion of flow fields in the vicinity of a plant by turbine wakes (plant generated turbulence) $[43,44]$, and finally, turbulence from the largest length scales of atmospheric flows (atmospheric turbulence). An estimate of $\tau_{0}$ for the three sources, and its comparison with duration out to which scalings persist, proves useful in determining the dominant source of fluctuations.

Using a representative value of $u_{\mathrm{rms}} \sim 4 \mathrm{~m} \mathrm{~s}^{-1}$ and $l_{0} \sim 1 \mathrm{~km}$ for the atmospheric boundary layer thickness [45], we estimate $\tau_{0}=l_{0} / u_{\text {rms }}=250 \mathrm{~s} \sim 4$ min. Plant generated turbulence would possess an integral scale $l_{0} \sim$ few kilometers, the plant's linear dimension. Taking a putative plant linear dimension of $5 \mathrm{~km}$ yields a large eddy turnover time estimate of $\tau_{0}=5000 \mathrm{~m} / 4 \mathrm{~m} \mathrm{~s}^{-1}=1250 \mathrm{~s} \sim$ tens of

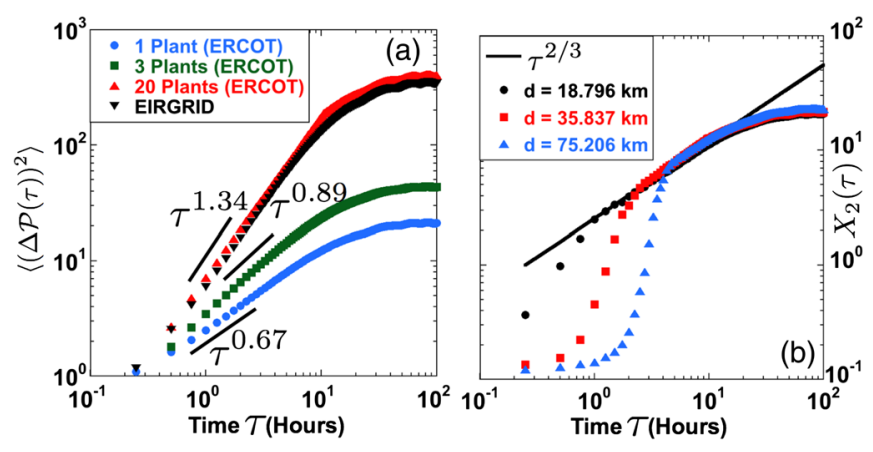

FIG. 2. (a) Aggregate power scaling $\langle[\Delta \mathcal{P}(\tau))] 2\rangle=A \tau^{\zeta}$ steepens with increasing number of plants $N$ from $\zeta=0.68 \pm 0.05$ ( $N=1$, solid blue circles) through $\zeta=0.89 \pm 0.05(N=3$, solid green squares) until scaling saturates at $\zeta=1.39 \pm 0.05$ ( $N=20$, solid red triangles) for ERCOT. EIRGRID data ( $N=224$, solid black inverted triangles) yields $\zeta=1.34 \pm 0.01$ in exact agreement with $\zeta=4 / 3$ bound. (b) $X_{2}(\tau)$ for interplant distance $d=18.796 \mathrm{~km}$ (solid black circles), $d=35.837 \mathrm{~km}$ (solid red squares) and $d=75.206 \mathrm{~km}$ (solid blue triangles) shows $X_{2}(\tau)=B \tau^{2 / 3}$ for $\tau>\tau_{d}$, where $\tau_{d}$ monotonically increases with $d$. All curves in (a) and (b) were normalized ( $A=B=1$ ) for easy comparison.

minutes. Atmospheric turbulence yields $\tau_{0} \sim 10 \mathrm{~h}$ as already estimated from Howard data. Estimates of $\tau_{0}$ for boundary layer dynamics (few minutes) and plant generated turbulence (tens of minutes) are much shorter than the $\sim 10 \mathrm{~h}$ duration up to which $\tau^{2 / 3}$ scaling persists in aggregate plant power fluctuations [10,14]. In Fig. 2(a), we plot the second order structure function of power fluctuations for a single wind plant $(N=1)$ in the ERCOT system (15 min sampling for $1 \mathrm{yr}$ duration, previously presented in Ref. [14]). There too, we see $\tau^{0.68 \pm 0.05}$ persists up to $\tau=11 \mathrm{~h}$.

Irrespective of the turbulent source that generates eddies, it is $\bar{u}$ and $u_{\text {rms }}$ of large scale atmospheric flows that transport eddies past the plant [39]. The presence of $u_{\mathrm{rms}}$ in the turbine spectrum (Eqs. (3) and (4)) assures plant generated turbulence and boundary layer dynamics are slave to the longer time scale dynamics of large scale atmospheric structures which contain the most energy. Aggregate plant power smooths over short time scales corresponding to fluctuation time scales of eddies of size $r \leq d$, where $d$ is interturbine spacing. However, over $D \sim$ hundreds of $\mathrm{km}$, a plant few kilometers in span behaves as an integral probe for all eddies larger than itself, since these large eddies strongly correlate all turbine outputs. As a result, one observes the $\tau^{2 / 3}$ spectral scaling extend from the turbine to the plant scale.

Moving from a single $(N=1)$ plant, we now consider grid level fluctuations in aggregate wind power $\mathcal{P}(t)=$ $\Sigma_{i=1}^{N} \Sigma_{j=1}^{M_{i}} P_{i j}(t)$ from several $(N>1)$ distributed wind plants, each with $M_{i}$ turbines. In Fig. 2(a), we plot the second order structure function $\left\langle[\Delta \mathcal{P}(\tau)]^{2}\right\rangle \equiv\langle[\mathcal{P}(t+\tau)-$ $\left.\mathcal{P}(t)]^{2}\right\rangle$ for composite wind power $\mathcal{P}(t)$ for $N=1,3$, and 
20 wind plants in the ERCOT system. The scaling $\left\langle[\Delta \mathcal{P}(\tau)]^{2}\right\rangle \sim \tau^{\zeta}$ steepens with increasing $N$ from the expected $\zeta=0.68 \pm 0.05 \quad(N=1) \quad$ through $\quad \zeta=$ $0.89 \pm 0.05(N=3)$ before saturating at $\zeta=1.39 \pm 0.05$ $(N=20)$. To emphasize the scaling saturation, we overlay observed scaling for data (15 min sampling over $5 \mathrm{yr}$ ) from the Irish grid (EIRGRID) [46] comprising $N=224$ plants, previously published in Ref. [3], which yields $\zeta=1.34 \pm 0.01$. Larger the value of $\zeta$ (steeper scaling of $\left\langle[\Delta \mathcal{P}(\tau)]^{2}\right\rangle$ ), greater the geographic smoothing magnitude. The fact that scaling stalls at $\zeta=4 / 3$ despite an order of magnitude increase in the number of wind plants from $N=20$ (ERCOT) to $N=224$ (EIRGRID) implies, there exists a limit to geographic smoothing.

Large scale atmospheric influence directly impacts geographic smoothing through instantaneous correlations in interplant power outputs. Since large scales convect small eddies nesting within them, two plants distance $d$ apart become correlated by eddies of size $d<r<l_{0}$ and exhibit correlated power fluctuations in the range of time scales $\tau_{d}<\tau<\tau_{0}$ and vanish for $\tau \leq \tau_{d}, \tau_{d}$ being the fluctuation time scale for eddy of size $r \equiv d$. From the above physical picture, we expect the second order cross-structure function of wind power defined as $X_{2}(\tau) \equiv\left\langle\left[P_{k}(t+\tau)-P_{l}(t)\right]^{2}\right\rangle$ between plant pairs $k$ and $l$ distance $d$ apart, will scale as $X_{2}(\tau) \sim \tau^{2 / 3}$ for $\tau>\tau_{d}$ and vanish for $\tau \leq \tau_{d}$. This expectation assumes the two wind plants are collinear along the streamwise direction of large scale flow and would only work if directional fluctuations are small and infrequent. Since ERCOT data did not contain wind direction information, we confirmed collinearity of plant separation with prevalent wind direction using monthly averages from land based stations through the NOAA database [47]. Figure 2(b) shows $X_{2}(\tau)$ scaling for three representative wind plant pairs in the ERCOT system where this expectation is met. A clear time scale $\left(\tau_{d}\right)$ is discernible below which no scaling exists, and above which $X_{2}(\tau) \sim \tau^{2 / 3}$ holds. Importantly, $\tau_{d}$ monotonically increases with interplant distance $d$, thus supporting our expectation.

We now use the above framework to explain the geographic smoothing mechanism and its $\tau^{4 / 3}$ spectral limit. Consider $N$ distributed wind plants, with interplant distance $d$ less than $(d<D)$, comparable to $(d \simeq D)$, or greater than $(d>D)$ spatial correlation length $D\left(l_{0} \simeq D\right)$. Summing all outputs into a composite signal $\mathcal{P}(t)$ representing the aggregate grid-scale wind power, we see $\mathcal{P}(t)=$ $\Sigma_{i=1}^{N} \Sigma_{j=1}^{M_{i}} P_{i j}(t)=K_{i j}\left[\overline{u_{i j}}{ }^{3}+3 \overline{u_{i j}} \tilde{u}_{i j}(t)+3 \overline{u_{i j}} \tilde{u_{i j}}(t)^{2}+\right.$ $\left.\tilde{u_{i j}}(t)^{3}\right]$. As odd functions, $\tilde{u_{i j}}(t)$ and $\tilde{u_{i j}}(t)^{3}$ take positive and negative values at different spatial locations for each instantaneous snapshot, with their sum approaching a small, nonzero value. However, $\tilde{u_{i j}}(t)^{2}$ being quadratic and always positive is amplified under summation $\Sigma_{i, j} \tilde{u}_{i j}(t)^{2}$ and exerts control over grid-scale fluctuations. Since the efficacy of geographic smoothing depends upon the decay of spatiotemporal correlations in $\mathcal{P}(t)$, we examine its temporal, two-point correlator given by

$$
\left.\mathcal{P}(t) \overline{\mathcal{P}_{(}} t+\tau\right)=\sum_{k, l} P_{k}(t) \overline{P_{k}}(t+\tau)+P_{k}(t) \overline{P_{l}}(t+\tau) .
$$

When individual plant outputs are summed, the crosscorrelation term (second term in rhs of Eq. (5) encoding long-range spatial correlations decays with increasing interplant distance and $\Sigma_{k, l} P_{k}(t) \overline{P_{l}}(t+\tau) \rightarrow 0$ as $d \rightarrow D$. This leaves only the self-correlation term $\left[\Sigma_{k, l} P_{k}(t) \hat{P_{k}^{-}}(t+\tau)\right]$, which represents true K41 scalings.

Whereas temporal sampling at an Eulerian point yields a non-Galilean invariant temporal spectrum for wind power, the temporal spectrum of a spatially averaged signal is Galilean invariant by default and respects K41 scalings [38]. Scaling up from $N=1$ towards several $(N>1)$ distributed plants spanning an area $\sim D^{2}$ feeding the grid, one approaches the asymptotic field averaged limit. In this limit, cross-correlations in Eq. (5) decay and the signal selfcorrelation is retained with true K41 scaling. Whereas the asymptotic convergence would occur as $\sqrt{N}$ if plants were independent, long range spatial correlation permits faster convergence as observed in Fig. 2(a). Consequently, gridscale aggregate power fluctuations $\left\langle[\Delta \mathcal{P}(\tau)]^{2}\right\rangle$ follow K41 dimensional scaling for $\tilde{u}(\tau)^{2}$, i.e. $\left\langle[\Delta \mathcal{P}(\tau)]^{2}\right\rangle \sim S_{2}^{2}(\tau) \sim \tau^{4 / 3}$ (Supplemental Material [16]). In fact, since one can never escape self-correlations, grid-scale fluctuations cannot smooth beyond this natural scaling limit; $\left\langle[\Delta \mathcal{P}(\tau)]^{2}\right\rangle \sim \tau^{\zeta}$ will steepen and $\zeta$ will increase from $\zeta=2 / 3$ for a single plant to $\zeta=4 / 3$ at the grid scale and stall there. Further increase in the number of wind plants $N$ cannot smooth fluctuations past this natural spectral bound as clearly evidenced from Fig. 2(a).

In summary, knowledge of the spectral bound and validation of its existence has immediate implications for wind engineering and policy alike. Once the spectral limit is hit, adding power from more plants located within the correlation distance $D$ does not change the spectrum. Adding power from plants situated beyond the correlation length will contribute more power to the zero frequency mode (mean power) and the spectrum will shift down, but will retain its $4 / 3$ spectral slope. Knowledge of this spectral limit helps estimate the ancillary reserves [48] needed on standby for a grid of given size and response time. The known spectral form, when fed as input to a stochastic load scheduling protocol [49], could potentially alleviate dynamic load balancing challenges. Similar spectral limits, if they exist, for other renewables such as solar [4], could inform policy by helping estimate the optimal energy mix for a regional grid with known set of resource constraints.

This work was supported by the Collective Interactions Unit, OIST Graduate University. M. M. B. thanks J. Apt for ERCOT and Howard data, EIRGRID for Irish data, and 
acknowledges discussions with J. Apt, N. Goldenfeld, S. Mandre, B. Fox-Kemper, I. Procaccia, S. Ciliberto, G. Falkovich, M. Chertkov, M. Venkadesan, N. Ouellette, and S. Kurien.

*bandi@ oist.jp

[1] D. J. C. MacKay, Sustainable Energy-Without the Hot Air (UIT Cambridge Ltd., Cambridge, England, 2009).

[2] J. Apt and P. Jaramillo, Variable Renewable Energy and the Electricity Grid (Taylor and Francis, London, 2014).

[3] G. Bel, C. P. Connaughton, M. Toots, and M. M. Bandi, New J. Phys. 18, 023015 (2016).

[4] K. Klima and J. Apt, Envir. Res. Lett. 10, 104001 (2015).

[5] R. Wiser, Z. Yang, M. Hand, O. Hohmeyer, D. Infield, P. H. Jensen, V. Nikolaev, M. O'Malley, G. Sinden, and A. Zervos, Wind Energy, In IPCC Special Report on Renewable Energy Sources and Climate Change Mitigation (Cambridge University Press, Cambridge, England and New York, USA, 2011).

[6] J. O. G. Tande, Appl. Energy 65, 395 (2000).

[7] C. Leuken, G. E. Cohen, and J. Apt, Environ. Sci. Technol. 46, 9761 (2012).

[8] M. A. Matos and R. J. Bessa, IEEE Transactions on Power Systems;IEEE Transactions on Power Electronics 26, 594 (2011).

[9] A. N. Kolmogorov, Dokl. Akad. Nauk SSSR 30, 299 (1941) [Sov. Phys. Dokl.].

[10] J. Apt, J. Power Sources 169, 369 (2007).

[11] P. Milan, M. Wächter, and J. Peinke, Phys. Rev. Lett. 110, 138701 (2013).

[12] R. Calif, R. Schmitt, and Y. Huang, Physica (Amsterdam) 392A, 4106 (2013).

[13] Whereas published results we cite are in frequency domain, to maintain consistency with our analysis, we quote all results in the time domain with the understanding that $\tau^{\zeta} \Leftrightarrow f^{-(\zeta+1)}$

[14] W. Katzenstein, E. Fertig, and J. Apt, Energy Policy 38, 4400 (2010)

[15] W. Katzenstein and J. Apt, Energy Policy 51, 233 (2012).

[16] See Supplemental Material at http://link.aps.org/ supplemental/10.1103/PhysRevLett.118.028301, for wind turbine power relation, turbine power conversion, K41 dimensional consideration, TH versus RSH and Galilean invariance breakdown, and Integral scale of atmospheric turbulence, which includes Refs. [17-34].

[17] E. Kulunk, in Fundamental and Advanced Topics in Wind Power, edited by R. Carriveau (Intech, 2011), Chap. 1.

[18] T. Burton, D. Sharpe, N. Jenkins, and E. Bossanyi, Wind Energy Handbook (John Wiley \& Sons Ltd., England, 2001).

[19] C. V. Moreno, H. A. Duarte, and J. U. Garcia, IEEE Trans. Energy Convers. 17, 267 (2002).

[20] A. Choukulkar, Y. Pichugina, C. T. M. Clack, R. Calhoun, R. Banta, A. Brewer, and M. Hardesty, Wind Energ., doi:10.1002/we.1929 (2015).
[21] D. A. Dutton and D. G. Deaven, in Some Properties of Atmospheric Turbulence. In Statistical Models and Turbulence. Lecture Notes in Physics, edited by M. Rosenblatt and C. W. Van Atta (Springer, New York, 1972), Vol. 12.

[22] R. H. Kraichnan, Phys. Fluids 7, 1723 (1964).

[23] H. Tennekes, J. Fluid Mech. 67, 561 (1975).

[24] S. Chen and R. H. Kraichnan, Phys. Fluids A 1, 2019 (1989).

[25] R. H. Kraichnan, Phys. Fluids 8, 575 (1965).

[26] U. Frisch, Turbulence: The Legacy of A. N. Kolmogorov (Cambridge University Press, Cambridge, England, 1995).

[27] W. D. McComb, Phys. Rev. E 71, 037301 (2005).

[28] A. A. Townsend, The Structure of Turbulent Shear Flow (Cambridge University Press; Cambridge, England, 1980), 2nd Edition.

[29] J. Counihan, Atmos. Environ. 9, 871 (1975).

[30] G. D. Nastrom, K. S. Gage, and W. H. Jasperson, Nature (London) 310, 36 (1984).

[31] S. Lovejoy, D. Schertzer, and J. D. Stanway, Phys. Rev. Lett. 86, 5200 (2001).

[32] J. F. Muzy, R. Baiile, and P. Poggi, Phys. Rev. E 81, 056308 (2010).

[33] E. Lindborg, J. Fluid Mech. 388, 259 (1999).

[34] F. A. Gifford, Agr. Forest Meteorol. 47, 155 (1989).

[35] C. W. Van Atta and J. C. Wyngaard, J. Fluid Mech. 72, 673 (1975).

[36] G. I. Taylor, Proc. R. Soc. A 164, 476 (1938).

[37] J. Mur-Amada and A. Boyad-Rujula, in Variability of Wind and Wind Power, Wind Power, edited by S. M. Muyeen (InTech, 2010), ISBN: 978-953-7619-81-7.

[38] M. Wilczek and R. J. A. M. Stevens, Wind Energ., doi:10.1002/we.1929 (2015).

[39] M. Wilczek, R. J. A. M. Stevens, and C. Meneveau, J. Fluid Mech. 769, R1 (2015).

[40] G. Giebel, (Ph.D. dissertation, Carl von Ossietsky, University of Oldenburg, 2000. Available at: http://www.drgiebel .de/GGiebel_DistributedWindEnergyInEurope.pdf).

[41] R. Steinberger-Willms, Ph.D. dissertation, Oldenburg University, 1993 (Verlag Shaker, Aachen, 1993), ISBN 3-86111-740-1, ISSN 0945-0726.

[42] L. Landberg, M. A. Hansen, K. Vesterager, and Bergstrøm, Report No. Ris $\varnothing-\mathrm{R}-929(\mathrm{EN})$ (Risoe National Laboratory, Roskilde, Denmark, 1997), ISBN 87-5502229-4.

[43] S. Frandsen, J. Wind Eng. Ind. Aerodyn. 39, 251 (1992).

[44] M. Calaf, C. Meneveau, and J. Meyers, Phys. Fluids 22, 015110 (2010).

[45] A. L. M. Grant, J. Atmos. Sci. 49, 226 (1992).

[46] http://www.eirgrid.com/operations/systemperformancedata/ windgeneration/.

[47] NOAA National Centers for Environmental Information, https://www.ncdc.noaa.gov.

[48] R. Doherty and M. O'Malley, IEEE Transactions on Power Systems; IEEE Transactions on Power Electronics 20, 587 (2005).

[49] P. A. Ruiz, C. R. Philbrick, and P. W. Sauer, Pow. Syst. Conf. and Expo. PSCE '09, doi:10.1109/PSCE. 2009.4840133 (2009). 